\title{
Minimality of dynamic input-output decoupling for nonlinear systems
}

\author{
H.J.C. Huijberts \\ Department of Mathematics and Computing Science, Eindhoven University of Technology, Eindhoven, Netherlands
}

\section{H. Nijmeijer}

Department of Applied Mathematics, University of Twente, Enschede, Netherlands

\section{L.L.M. van der Wegen}

School of Management Studies, University of Twente, Enschede, Netherlands

Received 23 November 1991

Revised 24 January 1992

\begin{abstract}
In this note we study the strong dynamic input-output decoupling problem for nonlinear systems. Using an algebraic theory for nonlinear control systems, we obtain for a dynamic input-output decouplable nonlinear system a compensator of minimal dimension that solves the decoupling problem.
\end{abstract}

AMS Subject Classifications: 93C10, 93B50, 93C35.

Keywords: Nonlinear control systems; input-output decoupling; dynamic feedback.

\section{Introduction}

Starting with Singh [17,19] an extensive literature has emerged on the (dynamic) input-output decoupling problem for nonlinear control systems, see e.g. [5,15,14,6,13]. The solution of this problem of designing a nonlinear dynamic compensator for a given nonlinear system so that the resulting compensated system is input-output decoupled is nowadays well-understood and in fact several different algorithms for constructing a decoupling compensator are known, see [17,19,5,15].

However, in contrast with the theory on linear decoupling (see [1]) some aspects of the nonlinear dynamic decoupling problem have not yet been addressed. Here we will focus on one such aspect; namely the construction of a decoupling compensator of minimal dimension (see [12] for a treatment of the linear case). Indeed, the algebraic framework of [4] allows us to develop a straightforward method for obtaining a minimal solution of the decoupling problem. Hereby we are able to exploit and further develop the theory about the so called 'essential orders' of a nonlinear system, as defined in [6]. The essential orders represent the smallest (algebraic) structure at infinity [14] which can be achieved by a suitably selected decoupling compensator (cf. [6]). As a result, we show that these essential orders may be computed directly from the here described dynamic compensator, which we call a Singh compensator. This work is partly based on our preliminary report [9] (see also [20] and [8]).

In [6] it was shown that the algorithm of [5] yields a decoupling compensator achieving the (algebraic) structure at infinity represented by the essential orders. This leads to the conjecture that the algorithm of 
[5] also yields a minimal order decoupling compensator. However, by the absence of applying regular static state feedback while performing the algorithm, the tools employed in this paper to establish minimality of the Singh compensator cannot immediately be used to establish the minimality of the algorithm of [5]. In [22] also a minimal order input-output decoupling algorithm was proposed. Basically this algorithm is a combination of the algorithms from [5] and [15]. However, the results of [22] are incomplete in that the authors could not correctly establish a proof of their result on minimality. Since the basic difference between the algorithms of [22] and [5] is that while performing the algorithm of [22] regular static state feedback is employed, it follows from the above discussion that probably the minimality of the algorithm of [22] can be established by employing the algebraic arguments of our present paper.

The paper is organized as follows. In Section 2 we introduce the algebraic theory for nonlinear control systems. The material in this section is taken from [4]. In Section 3 we introduce the dynamic input-output decoupling problem and obtain a compensator of minimal order that solves the problem. Section 4 contains the conclusions.

\section{Algebraic tools}

We first introduce an algebraic theory for nonlinear control systems. This material is taken (almost verbatim) from [4].

Consider a square analytic system of the form

$$
\begin{aligned}
& \dot{x}=f(x)+g(x) u, \\
& y=h(x)
\end{aligned}
$$

where $x=\left(x_{1}, \ldots, x_{n}\right)^{\mathbf{T}} \in \mathbb{R}^{n}$ are local coordinates for the state space manifold $M, u \in \mathbb{R}^{m}, y \in \mathbb{R}^{m}$, $g(x)=\left(g_{1}(x) \cdots g_{m}(x)\right), h(x)=\left(h_{1}(x), \ldots, h_{m}(x)\right)^{\mathrm{T}}, f, g_{1}, \ldots, g_{m}$ are analytic vector fields on $M$, and $h_{1}, \ldots, h_{m}$ are analytic functions on $M$.

Recall that a meromorphic function $\eta$ is a function of the form $\eta=\pi / \theta$, where $\pi$ and $\theta$ are analytic functions. Assume that the control functions $u(t)$ are $n$ times continuously differentiable. Then define: $u^{0}:=u(t), u^{(i+1)}:=(\mathrm{d} / \mathrm{d} t) u^{(i)}(t)$. View $x, u, \ldots, u^{(n-1)}$ as variables and let $\mathscr{K}$ denote the field consisting of the set of rational functions of $\left(u, \ldots, u^{(n-1)}\right)$ with coefficients that are meromorphic in $x$. For the system (1) we define in a natural way

$$
\begin{aligned}
& \dot{y}=\dot{y}(x, u)=\frac{\partial h}{\partial x}[f(x)+g(x) u] \\
& y^{(k+1)}=y^{(k+1)}\left(x, u, \ldots, u^{(k)}\right)=\frac{\partial y^{(k)}}{\partial x}[f(x)+g(x) u]+\sum_{i=0}^{k-1} \frac{\partial y^{(k)}}{\partial u^{(i)}} u^{(i+1)} .
\end{aligned}
$$

Note that $\dot{y}, \ldots, y^{(n)}$ so defined have components in the field $\mathscr{K}$.

Let $\mathscr{E}$ denote the vector space (over $\mathscr{K}$ ) spanned by $\left\{\mathrm{d} x, \mathrm{~d} u, \ldots, \mathrm{d} u^{(n-1)}\right\}$. We introduce the chain of subspaces $\mathscr{E}_{0} \subset \cdots \subset \mathscr{E}_{n}$ of $\mathscr{E}$ by

$$
\mathscr{E}_{k}:=\operatorname{span}\left\{\mathrm{d} x, \mathrm{~d} \dot{y}, \ldots, \mathrm{d} y^{(k)}\right\} .
$$

Note that in (3) the span is taken with respect to the field $\mathscr{K}$ and not with respect to the real numbers.

A convenient way for performing the necessary calculations required in the algebraic theory is provided by the so called Singh's algorithm. Singh's algorithm has been introduced in [18] for calculation of the left inverse of a nonlinear system. It is a generalization of the algorithm from [7], which was only applicable under some restrictive assumptions. The version of Singh's algorithm presented here is taken from [4]. 
Algorithm 2.1. Singh's algorithm. Consider the analytic nonlinear control system (1).

Step 1. Calculate

$$
\dot{y}=\frac{\partial h}{\partial x}[f(x)+g(x) u]=: a_{1}(x)+b_{1}(x) u
$$

and define

$$
\rho_{1}=s_{1}:=\operatorname{rank} b_{1}(x)
$$

where the rank is taken over the field of meromorphic functions of $x$. Permute, if necessary, the components of the output so that the first $\rho_{1}$ rows of $b_{1}(x)$ are linearly independent. Decompose $y$ according to $\dot{y}=\left(\dot{\tilde{y}}_{1}^{\mathrm{T}} \hat{\hat{y}}_{1}^{\mathrm{T}}\right)^{\mathrm{T}}$, where $\dot{\tilde{y}}_{1}$ consists of the first $\rho_{1}$ rows of $\dot{y}$. Since the last rows of $b_{1}(x)$ are linearly dependent on the first $\rho_{1}$ rows, we can write

$$
\dot{\tilde{y}}_{1}=\tilde{a}_{1}(x)+\tilde{b}_{1}(x) u, \quad \dot{\hat{y}}_{1}=\dot{\hat{y}}_{1}\left(x, \dot{\tilde{y}}_{1}\right)
$$

where the last equation is affine in $\dot{\tilde{y}}_{1}$. Finally, set $\tilde{B}_{1}(x):=\tilde{b}_{1}(x)$.

Step $k+1$. Suppose that in Steps 1 through $k, \tilde{\tilde{y}}_{1}, \ldots, \tilde{y}_{k}^{(k)}, \hat{y}_{k}^{(k)}$ have been defined so that

$$
\begin{aligned}
& \dot{\bar{y}}_{1}=\tilde{a}_{1}(x)+\tilde{b}_{1}(x) u, \\
& \quad \vdots \\
& \tilde{y}_{k}^{(k)}=\tilde{a}_{k}\left(x,\left\{\tilde{y}_{i}^{(j)} \mid 1 \leqslant i \leqslant k-1, i \leqslant j \leqslant k\right\}\right)+\tilde{b}_{k}\left(x,\left\{\tilde{y}_{i}^{(j)} \mid 1 \leqslant i \leqslant k-1, i \leqslant j \leqslant k-1\right\}\right) u, \\
& \hat{y}_{k}^{(k)}=\hat{y}_{k}^{(k)}\left(x,\left\{\tilde{y}_{i}^{(j)} \mid 1 \leqslant i \leqslant k, i \leqslant j \leqslant k\right\}\right)
\end{aligned}
$$

and so that they are rational functions of $\tilde{y}_{i}^{(j)}$ with coefficients in the field of meromorphic functions of $x$. Suppose also that the matrix $\tilde{B}_{k}:=\left[\tilde{b}_{1}^{\mathrm{T}}, \ldots, \tilde{b}_{k}^{\mathrm{T}}\right]^{\mathrm{T}}$ has full rank equal to $\rho_{k}$, where the rank is taken with respect to the field of rational functions of $\left\{\tilde{y}_{i}^{(j)} \mid 1 \leqslant i \leqslant k-1, i \leqslant j \leqslant k-1\right\}$ with coefficients in the field of meromorphic functions of $x$. Then calculate

$$
\hat{y}_{k}^{(k+1)}=\frac{\partial}{\partial x} \hat{y}_{k}^{(k)}[f(x)+g(x) u]+\sum_{i=1}^{k} \sum_{j=i}^{k} \frac{\partial \hat{y}_{k}^{(k)}}{\partial \tilde{y}_{i}^{(j)}} \tilde{y}_{i}^{(j+1)}
$$

and write this as

$$
\hat{y}_{k}^{(k+1)}=a_{k+1}\left(x,\left\{\tilde{y}_{i}^{(j)} \mid 1 \leqslant i \leqslant k, i \leqslant j \leqslant k+1\right\}\right)+b_{k+1}\left(x,\left\{\tilde{y}_{i}^{(j)} \mid 1 \leqslant i \leqslant k, i \leqslant j \leqslant k\right\}\right) u .
$$

Define $B_{k+1}:=\left[\tilde{B}_{k}^{\mathrm{T}}, b_{k+1}^{\mathrm{T}}\right]^{\mathrm{T}}$, and $\rho_{k+1}:=$ rank $B_{k+1}$ where the rank is taken with respect to the field of rational functions of $\left\{\tilde{y}_{i}^{(j)} \mid 1 \leqslant i \leqslant k, i \leqslant j \leqslant k\right\}$ with coefficients in the field of meromorphic functions of $x$. Permute, if necessary, the components of $\hat{y}_{k}^{(k+1)}$ so that the first $\rho_{k+1}$ rows of $B_{k+1}$ are linearly independent. Decompose $\hat{y}_{k}^{(k+1)}$ as $\hat{y}_{k}^{(k+1)}=\left(\tilde{y}_{k+1}^{(k+1)^{\mathrm{T}}} \hat{y}_{k+1}^{(k+1)^{\mathrm{T}}}\right)^{\mathrm{T}}$, where $\tilde{y}_{k+1}^{(k+1)}$ consists of the first $s_{k+1}:=\left(\rho_{k+1}-\rho_{k}\right)$ rows. Since the last rows of $B_{k+1}$ are linearly dependent on the first $\rho_{k+1}$ rows, we can write

$$
\begin{aligned}
& \dot{\tilde{y}}_{1}=\tilde{a}_{1}(x)+\tilde{b}_{1}(x) u, \\
& \quad \vdots \\
& \tilde{y}_{k+1}^{(k+1)}-\tilde{a}_{k+1}\left(x,\left\{\tilde{y}_{i}^{(j)} \mid 1 \leqslant i \leqslant k, i \leqslant j \leqslant k+1\right\}\right)+\tilde{b}_{k+1}\left(x,\left\{\tilde{y}_{i}^{(j)} \mid 1 \leqslant i \leqslant k, i \leqslant j \leqslant k\right\}\right) u, \\
& \hat{y}_{k+1}^{(k+1)}=\hat{y}_{k+1}^{(k+1)}\left(x,\left\{\tilde{y}_{i}^{(j)} \mid 1 \leqslant i \leqslant k+1, i \leqslant j \leqslant k+1\right\}\right)
\end{aligned}
$$

where once again everything is.rational in $\tilde{y}_{i}^{(j)}$. Finally, set $\tilde{B}_{k+1}:=\left[\tilde{B}_{k}^{\mathrm{T}}, \tilde{b}_{k+1}^{\mathrm{T}}\right]^{\mathrm{T}}$. 
The number $\rho^{*}=\rho_{n}$ is called the rank of (1).

Then we have the following result (cf. Di Benedetto et al. [4]).

Theorem 2.2. Consider the nonlinear system (1) and apply Singh's algorithm to it. Then for each $1 \leqslant k \leqslant n$,

(i) $\left\{\mathrm{d} x,\left\{\mathrm{~d} \tilde{y}_{i}^{(j)} \mid 1 \leqslant i \leqslant k, i \leqslant j \leqslant k\right\}\right\}$ is a basis for $\mathscr{E}_{k}$,

(ii) $\operatorname{dim}_{\not{T}} \mathscr{E}_{k}=n+\rho_{1}+\cdots+\rho_{k}$.

Using the algebraic theory presented above we define what we mean by a regular dynamic state feedback (cf. [4]).

Definition 2.3. Consider a nonlinear system (1), together with a dynamic state feedback

$$
\begin{aligned}
& \dot{z}=\alpha(x, z)+\beta(x, z) v^{\prime}, \\
& u=\gamma(x, z)+\delta(x, z) v
\end{aligned}
$$

with $v$ denoting the new controls and $\alpha, \beta, \gamma, \delta$ analytic functions of $x$ and $z$. (11) is called a regular dynamic state feedback for (1) if the system

$$
\begin{aligned}
& \dot{x}=f(x)+g(x) u, \\
& \dot{z}=\alpha(x, z)+\beta(x, z) v, \\
& u=\gamma(x, z)+\delta(x, z) v
\end{aligned}
$$

with controls $v^{\prime}$ and outputs $u$ has full rank (equal to $m$ ).

For later use we associate a notion of regularity with Singh's algorithm in the following way (see [3] for a related, but somewhat different notion of regularity).

Definition 2.4. Consider the nonlinear system (1) and let a point $x_{0} \in M$ be given.

(i) We call $x_{0}$ a regular point for (1) if for an appropriate application of Singh's algorithm to (1) there exists a point $\left\{\tilde{y}_{i 0}^{(j)} \mid 1 \leqslant i \leqslant n-1, i \leqslant j \leqslant n-1\right\}$ such that

$$
\operatorname{rank}_{\mathbb{R}} \tilde{B}_{k}\left(x_{0},\left\{\tilde{y}_{i 0}^{(j)} \mid 1 \leqslant i \leqslant k-1, i \leqslant j \leqslant k-1\right\}\right)=\rho_{k} \quad(k=1, \ldots, n) .
$$

(ii) We call $x_{0}$ a strongly regular point for (1) if for each application of Singh's algorithm to (1) there exists a point $\left\{\tilde{y}_{i 0}^{(j)} \mid 1 \leqslant i \leqslant n-1, i \leqslant j \leqslant n-1\right\}$ such that (13) holds.

\section{Minimality of dynamic input-output decoupling}

In this section we study the dynamic input-output decoupling problem. We study a strong version of this problem. For a nonlinear system (1), define the relative degrees $r_{i}(i=1, \ldots)$ as the smallest $k \in \mathbb{N}$ for which

$$
\frac{\partial y_{i}^{(k)}}{\partial u} \not \equiv 0 .
$$

If all relative degrees are finite define the decoupling matrix $A(x)$ with entries

$$
a_{i j}(x)=\frac{\partial y_{i}^{\left(r_{i}\right)}}{\partial u_{j}}(x) \text {. }
$$

A system (1) is said to be input-output decoupled if each of its inputs influences one and only one of its 
outputs. The system is said to be strongly input-output decoupled if all relative degrees are finite, its decoupling matrix is an invertible diagonal matrix, and

$$
\frac{\partial y_{i}^{(k)}}{\partial u_{j}}=0\left(i=1, \ldots, m ; j \neq i ; k \geqslant r_{i}+1\right) .
$$

Remark 3.5. Note that a strongly input-output decoupled system is input-output decoupled. The converse does not need to hold (see [16] for details).

Definition 3.6. Consider a nonlinear system (1) and let $x_{0}$ be given. Then the strong dynamic input-output decoupling problem via regular dynamic state feedback (SDIODP) is said to be solvable around $x_{0}$ if there exist an integer $\nu$, a dynamic state feedback on $\mathbb{R}^{\nu}$ of the form (11), a neighborhood $U \subset M$ of $x_{0}$ and an open subset $\mathscr{Z}$ of $\mathbb{R}^{\nu}$ such that the system $(1,11)$ restricted to $U \times \mathscr{Z}$ is strongly input-output decoupled.

In [4] it was shown that if $x_{0}$ is a (strongly) regular point for (1), the dynamic input-output decoupling problem is solvable around $x_{0}$ if and only if $\rho^{*}=m$ (see also [5,15]).

We now present a special sort of regular dynamic state feedback that solves SDIODP around strongly regular points $x_{0}$ for (1). This dynamic state feedback is obtained via Singh's algorithm and we call it a Singh compensator. The Singh compensator is obtained as follows. Consider the nonlinear system (1) and let $x_{0} \in M$ be a strongly regular point for (1). Furthermore assume that $\rho^{*}=m$. Apply Singh's algorithm to (1). This yields at the $n$-th step

$$
\dot{\tilde{Y}}_{n}=\tilde{A}_{n}\left(x,\left\{\tilde{y}_{i}^{(j)} \mid 1 \leqslant i \leqslant n-1, i \leqslant j \leqslant n\right\}\right)+\tilde{B}_{n}\left(x,\left\{\tilde{y}_{i}^{(j)} \mid 1 \leqslant i \leqslant n-1, i \leqslant j \leqslant n-1\right\}\right) u
$$

where

$$
\tilde{Y}_{n}=\left(\tilde{y}_{1}^{\mathrm{T}} \cdots \bar{y}_{n}^{(n-1)^{\mathrm{T}}}\right)^{\mathrm{T}}
$$

and

$$
\tilde{A}_{n}\left(x,\left\{\tilde{y}_{i}^{(j)} \mid 1 \leqslant i \leqslant n-1, i \leqslant j \leqslant n\right\}\right)=\left(\begin{array}{c}
\tilde{a}_{1}(x) \\
\tilde{a}_{2}\left(x, \dot{\bar{y}}_{1}, \tilde{y}_{i}^{(2)}\right) \\
\vdots \\
\tilde{a}_{n}\left(x,\left\{\tilde{y}_{i}^{(j)} \mid 1 \leqslant i \leqslant n-1, i \leqslant j \leqslant n\right\}\right)
\end{array}\right) .
$$

Moreover we know that there exist a neighborhood $U \subset M$ of $x_{0}$, a point $\left(\tilde{y}_{i 0}^{(j)} \mid 1 \leqslant i \leqslant n-1, i \leqslant j \leqslant n\right)$ and a neighborhood $Y_{0}$ of this point such that $\tilde{B}_{n}$ is invertible on $U \times Y_{0}$. Then on $U \times Y_{0}(17)$ yields in particular

$$
u=\tilde{B}_{n}^{-1}\left[\dot{\hat{Y}}_{n}-\tilde{A_{n}}\right] .
$$

For $i=1, \ldots, m$, let $\gamma_{i}$ be the lowest time-derivative and $\delta_{i}$ be the highest time-derivative of $y_{i}$ appearing in (18). Then we can rewrite (18) as

$$
\begin{aligned}
u= & \phi_{1}\left(x,\left\{y_{i}^{(j)} \mid 1 \leqslant i \leqslant m, \gamma_{i} \leqslant j \leqslant \delta_{i}-1\right\}\right) \\
& +\sum_{i=1}^{m} \phi_{2 i}\left(x,\left\{y_{i}^{(j)} \mid 1 \leqslant i \leqslant m, \gamma_{i} \leqslant j \leqslant \delta_{i}-1\right\}\right) y_{i}^{\left(\delta_{i}\right)}
\end{aligned}
$$

for certain vector-valued functions $\phi_{1}, \phi_{2 i}(i=1, \ldots, m)$. 
Let $z_{i}(i=1, \ldots, m)$ be a vector of dimensional $\delta_{i}-\gamma_{i}$ and consider the system

$$
\begin{aligned}
& \dot{z}_{i}=A_{i} z_{i}+B_{i} v_{i} \quad(i=1, \ldots, m), \\
& u=\phi_{1}\left(x, z_{1}, \ldots, z_{m}\right)+\sum_{i=1}^{m} \phi_{2 i}\left(x, z_{1}, \ldots, z_{m}\right) v_{i}
\end{aligned}
$$

with $\left(A_{i}, B_{i}\right)$ in Brunovsky canonical form. Then (20) is called a Singh compensator for (1) around $x_{0}$.

Remark 3.7. (i) In [10] it was shown that a Singh compensator is indeed a regular dynamic state feedback.

(ii) Note that the Singh compensator (20) in general will be a meromorphic feedback on an open subset $U \times \mathscr{Z} \subset M \times \mathbb{R}^{\sigma}$. Hence we can always find open subsets $\bar{U} \subset U$ and $\overline{\mathscr{Z}} \subset \mathscr{Z}$ such that (20) defined on $\bar{U} \times \overline{\mathscr{Z}}$ is analytic.

Inspection of Singh's algorithm and the construction of a Singh compensator (20) gives that for the compensated system (1), (20) we have that $y_{i}, \ldots, y_{i}^{\left(\delta_{i}-1\right)}$ are independent of the new controls and that $y_{i}^{\left(\delta_{i}\right)}=v_{i}(i=1, \ldots, m)$. Thus the decoupling matrix of (1), (20) is given by $A(x)=I_{m}$, and (16) holds. Hence any Singh compensator (20) around a strongly regular point $x_{0}$ solves the SDIODP around $x_{0}$.

We now prove that a Singh compensator is a decoupling precompensator of minimal order. The proof consists of two parts: we first prove that any Singh compensator around a strongly regular point $x_{0}$ has the same dimension, to be denoted by $\sigma$. Then we prove that for any dynamic decoupling feedback (11) with a $\nu$-dimensional state space we have that $\nu \geqslant \sigma$.

So consider the system (1) and let $x_{0}$ be a strongly regular point for (1). First assume that we apply Singh's algorithm to (1) in one specific way. Denote the dimension of the Singh compensator for (1) around $x_{0}$ obtained in this way by $\sigma$. Then obviously $\sigma=\sum_{i=1}^{m}\left(\delta_{i}-\gamma_{i}\right)$. Next note that $\gamma_{i}$ is the smallest $k \in \mathbb{N}$ for which $y_{i}$ is an entry of $\tilde{y}_{k}$. Inspection of Singh's algorithm gives that the set $\left\{y_{i} \mid \gamma_{i}=k\right\}$ has $s_{k}$ elements. Therefore,

$$
\sum_{i=1}^{m} \gamma_{i}=\sum_{k=1}^{n} k s_{k}
$$

Given the fact that $s_{1}=\rho_{1}$ and $s_{k}=\rho_{k}-\rho_{k-1}(k=2, \ldots, n), s_{1}, \ldots, s_{n}$ may be defined intrinsically (i.e. independent of Singh's algorithm, see Theorem 2.2). Hence we see from (21) that $\sum_{i=1}^{m} \gamma_{i}$ is independent of the specific way we apply Singh's algorithm. Next we show that the $\delta_{i}(i=1, \ldots, m)$ are also intrinsically defined. For this we need:

Definition 3.8 [2]. Let $V$ be a given vector space over a field $\mathscr{F}$. Let $A=\left\{\lambda_{1}, \ldots, \lambda_{r}\right\}$ be a family of vectors in $V$. Then $\lambda_{i}$ is called an essential vector of $\Lambda$ if

$$
\nexists \alpha_{1}, \ldots, \alpha_{i-1}, \alpha_{i+1}, \ldots, \alpha_{r} \in \mathscr{F}: \lambda_{i}=\sum_{j \neq i} \alpha_{j} \lambda_{j}
$$

Using simple arguments from linear algebra we can prove:

Lemma 3.9. Let $V$ be a given vector space over a field $\mathscr{F}$. Let $\Lambda=\left\{\lambda_{1}, \ldots, \lambda_{r}\right\}$ be a family of vectors in $V$. Let $s:=\operatorname{dim} \operatorname{span}\left\{\lambda_{1}, \ldots, \lambda_{r}\right\}$ and assume that $\left\{\lambda_{1}, \ldots, \lambda_{s}\right\}$ is a set of linearly independent vectors. Then $\lambda_{i}$ $(i=1, \ldots, s)$ is an essential vector of $\Lambda$ if and only if for all $j=s+1, \ldots, r$,

$$
\lambda_{j}=\sum_{k=1}^{s} \alpha_{j k} \lambda_{k} \Rightarrow \alpha_{j i}+0
$$


Definition 3.10 [6]. Consider a nonlinear system (1) satisfying $\rho^{*}=m$. The essential order $\varepsilon_{i}$ of the output $y_{i}$ of (1) is defined as the smallest $k \in\{1, \ldots, n\}$ for which $\mathrm{d} y_{i}^{(k)}$ is an essential vector of $\mathscr{E}_{n}$.

Remark 3.11. The relative degrees and essential orders as defined in Definition 3.10 are well-defined, since $\rho^{*}=m$ (cf. [6]).

Lemma 3.12. Consider the nonlinear system (1) satisfying $\rho^{*}=m$ and apply Singh's algorithm to it. Then for $i=1, \ldots, m: \delta_{i}=\varepsilon_{i}$.

Proof. By definition of the essential orders and Theorem 2.2 we have that $\mathrm{d} y_{i}^{(k)}$ is not an essential vector of $\mathscr{E}_{k}$ for $k=1, \ldots, \varepsilon_{i}-1$. This implies by Lemma 3.9 that $\partial \hat{y}_{\varepsilon_{i}-1}^{\left(\varepsilon_{i}-1\right)} / \partial y_{i}^{\left(\varepsilon_{i}-1\right)} \neq 0$ and hence $\delta_{i} \geqslant \varepsilon_{i}$. Moreover, by the definition of the essential orders and Theorem 2.2 we have that $\mathrm{d} y_{i}^{\left(\varepsilon_{i}\right)}$ is an essential vector of $\mathscr{E}_{k}$ for $k=\varepsilon_{i}, \ldots, n$. Again by Lemma 3.9 this implies that $\partial \hat{y}_{k}^{(k)} / \partial y_{i}^{(r)}=0$ for $k=\varepsilon_{i}, \ldots, n$ and $r=\varepsilon_{i}, \ldots, n$. This means that $\delta_{i} \leqslant \varepsilon_{i}$. Hence $\delta_{i}=\varepsilon_{i}$.

Remark 3.13. Note that for determining the essential orders we do not have to use Definition 3.10, by the result of Lemma 3.12 we could use Singh's algorithm for doing this.

We have now proved:

Proposition 3.14. Consider a nonlinear system (1) satisfying $\rho^{*}=m$ and let $x_{0}$ be a strongly regular point for (1). Then every Singh compensator for (1) around $x_{0}$ has dimension

$$
\sigma=\sum_{i=1}^{m} \varepsilon_{i}-\sum_{k=1}^{n} k s_{k} .
$$

Our main result can be stated as follows:

Theorem 3.15. Consider a nonlinear system (1) satisfying $\rho^{*}=m$. Let $x_{0}$ be a strongly regular point for (1). Then every Singh compensator for (1) around $x_{0}$ is a dynamic state feedback of minimal order solving the SDIODP for (1) around $x_{0}$.

For the proof of Theorem 3.15 we need some technical lemmas. Before stating these lemmas, we introduce some notation. Consider a (square) nonlinear system (1) together with a dynamic state feedback (11). For (1), (11), let $\mathscr{K}^{\mathrm{e}}$ denote the field consisting of the set of rational functions of $\left(v, \ldots, v^{(n+\nu-1)}\right)$ with coefficients that are meromorphic in $x$ and $z$. Let

$$
\mathscr{E}_{k}^{e}:=\operatorname{span}_{\mathscr{F}^{\mathrm{e}}}\left\{\mathrm{d} x, \mathrm{~d} z, \mathrm{~d} \dot{y}, \ldots, \mathrm{d} y^{(k)}\right\} \quad(k=1, \ldots, n+\nu-1) .
$$

Furthermore, denote the relative degrees of (1), (11) by $r_{1}^{\mathrm{e}}, \ldots, r_{m}^{\mathrm{e}}$ and its essential orders by $\varepsilon_{1}^{\mathrm{e}}, \ldots, \varepsilon_{m}^{\mathrm{e}}$.

Lemma 3.16. Consider a square nonlinear system (1) of full rank. Then

(i) $\varepsilon_{i}, r_{i}<+\infty \quad(i=1, \ldots, m)$,

(ii) $\varepsilon_{i} \geqslant r_{i} \quad(i=1, \ldots, m)$,

(iii) $\varepsilon_{i}=r_{i}$; for all $i \in\{1, \ldots, m\}$ if and only if the SDIODP is locally solvable around a point $x_{0} \in M$ via a regular static state feedback (i.e., $\nu=0$ in (11)).

Proof. See [6].

Lemma 3.17. If for (1), $\mathrm{d} y_{i}^{(k)}$ is not an essential vector of $\mathscr{E}_{n}$, then for (1), (11), $\mathrm{d} y_{i}^{(k)}$ is not an essential vector of $\mathscr{E}_{n}^{\mathrm{e}}$. Hence $\varepsilon_{i}^{\mathrm{e}} \geqslant \varepsilon_{i}(i=1, \ldots, m)$. 
Proof. See Lemma 2 of [6].

Proposition 3.18. Consider the square nonlinear system (1). Let $x_{0}$ be a strongly regular point for (1) and consider a regular dynamic state feedback (11) of dimension $\nu$ that solves the SDIODP around $x_{0}$. Then $\nu \geqslant \sigma$.

Proof. Let $x_{0} \in M$ be a strongly regular point for (1) and consider a regular dynamic state feedback (11) that solves the SDIODP around $x_{0}$. Then by Lemma 3.16 and Lemma 3.17 we have that $\varepsilon_{i}^{\mathrm{c}}=r_{i}^{\mathrm{c}} \geqslant \varepsilon_{i}$ $(i=1, \ldots, m)$. It is well known that for (1), (11) the differentials $\mathrm{d} y_{i}^{(k)}(x, z)\left(i=1, \ldots, m ; j=0, \ldots, r_{i}^{\mathrm{e}}\right)$ are linearly independent (over $\overline{\mathscr{K}}^{\mathrm{e}}$, the subfield of $\mathscr{K}^{\mathrm{e}}$ consisting of the meromorphic functions of $x$ and $z$ ), cf. $[13,16]$. By Theorem 2.2 and the proof of Proposition 3.14 we can find a reordering of the outputs of (1) and integers $\gamma_{1}, \ldots, \gamma_{m}$ satisfying $\sum_{i=1}^{m} \gamma_{i}=\sum_{i=1}^{m} \varepsilon_{i}-\sigma$, such that for (1) the differentials $\left\{\mathrm{d} x,\left\{\mathrm{~d} y_{i}^{(j)} \mid 1 \leqslant i \leqslant m, \gamma_{i} \leqslant j \leqslant \varepsilon_{i}-1\right\}\right\}$ are linearly independent over $\mathscr{K}$. Assume that for (1), (11) these differentials are not linearly independent over $\overline{\mathscr{K}}^{\mathrm{e}}$. This implies that we can find $r \in\{1, \ldots, m\}, s \in \mathbb{N}$ and a function

$$
\phi_{r s}\left(x,\left\{u_{i}^{(j)} \mid i \neq r, 0 \leqslant j \leqslant s\right\},\left\{u_{r}^{(j)} \mid 0 \leqslant j \leqslant s-1\right\}\right)
$$

such that for (1), (11),

$$
u_{r}^{(s)}=\phi_{r s}\left(x,\left\{u_{i}^{(j)} \mid i \neq r, 0 \leqslant j \leqslant s\right\},\left\{u_{r}^{(j)} \mid 0 \leqslant j \leqslant s-1\right\}\right) .
$$

By the proof of Lemma 3.3 in [4] this means that for all $k \geqslant s$ there exists a function

$$
\phi_{r k}\left(x,\left\{u_{i}^{(j)} \mid i \neq r, 0 \leqslant j \leqslant k\right\},\left\{u_{r}^{(j)} \mid 0 \leqslant j \leqslant s-1\right\}\right)
$$

such that

$$
u_{r}^{(k)}=\phi_{r k}\left(x,\left\{u_{i}^{(j)} \mid i \neq r, 0 \leqslant j \leqslant k\right\},\left\{u_{r}^{(j)} \mid 0 \leqslant j \leqslant s-1\right\}\right) .
$$

This implies that if we apply Singh's algorithm to (12) we find that $\rho_{n+\nu}<m$. Then by Definition 2.3 (11) is not a regular dynamic state feedback, which gives a contradiction. Hence for (1), (11) the differentials $\left\{\mathrm{d} x,\left\{\mathrm{~d} y_{i}^{(j)} \mid 1 \leqslant i \leqslant m, \gamma_{i} \leqslant j \leqslant \varepsilon_{i}-1\right\}\right\}$ are linearly independent over $\overline{\mathscr{H}}^{c}$. In particular this implies that

$$
\operatorname{rank} \overline{\not ̆}^{0}\left(\frac{\partial y_{i}^{(j)}}{\partial z}\right)_{1 \leqslant i \leqslant m, \gamma_{i} \leqslant j \leqslant \varepsilon_{i}-1}=\sum_{i=1}^{m}\left(\varepsilon_{i}-\gamma_{i}\right)=\sigma .
$$

Obviously,

$$
\operatorname{rank}_{\overline{\mathscr{H}}^{*}}\left(\frac{\partial y_{i}^{(i)}}{\partial z}\right)_{1 \leqslant i \leqslant m, \gamma_{i} \leqslant j \leqslant \varepsilon_{i}-1} \leqslant \operatorname{dim} z
$$

and so $\nu \geqslant \sigma$, which establishes our claim.

Theorem 3.15 is now an immediate consequence of Proposition 3.18.

Remark 3.19. In line with [6] it seems likely that the algorithm from [5] also yields a minimal decoupling compensator (as was also claimed - but incorrectly proved - in [22] for a combination of the algorithms from [5] and [15]). However, since the algorithm from [5] differs in a basic sense from the Singh algorithm as no static state feedbacks are allowed in each step of the algorithm (compare with equations (4) and (6)) it is not clear if the minimality result will hold for the algorithm from [5]. This is of course also not clear for the algorithms from [22] and [15]. 


\section{Conclusions}

Using algebraic tools we have obtained a compensator of minimal dimension that solves the strong dynamic input-output decoupling problem via regular dynamic state feedback for nonlinear systems. The proof of minimality of the compensator also provides further insight in the algebraic structure of nonlinear control systems.

\section{References}

[1] C. Commault, J. Descusse, J.M. Dion, J.F. Lafay and M. Malabre, About new decoupling invariants: the essential orders, Internat. J. Control 44 (1986) 689-700.

[2] M. Cremer, A precompensator of minimal order for decoupling a linear multivariable system, Internat. J. Control 14 (1971) 1089-1103.

[3] M.D. Di Benedetto and J.W. Grizzle, Intrinsic notions of regularity for local inversion, output nulling and dynamic extension of nonsquare systems, C-TAT 6 (1990) 357-381.

[4] M.D. Di Benedetto, J.W. Grizzle and C.H. Moog, Rank invariants of nonlinear systems, SIAM J. Control Optim. 27 (1989) $658-672$.

[5] J. Descusse and C.H. Moog, Decoupling with dynamic compensation for strong invertibility affine nonlinear systems, Internat. J. Control 42 (1985) 1387-1398.

[6] A. Glumineau and C.H. Moog, The essential orders and the nonlinear decoupling problem, Internat. J. Control 50 (1989) $1825-1834$.

[7] R.M. Hirschorn, Invertibility of multivariable nonlinear control systems, IEEE Trans. Automat. Control 24 (1979) $855-865$.

[8] H.J.C. Huijberts, Dynamic feedback in nonlinear synthesis problems, Ph.D. Thesis, University of Twente, 1991.

[9] H.J.C. Huijberts, H. Nijmeijer and L.L.M. van der Wegen, Dynamic disturbance decoupling for nonlinear systems, Memo No. 835, University of Twente, 1989. To appear in SLAM J. Control Optim.

[10] H.J.C. Huijberts, H. Nijmeijer and L.L.M. van der Wegen, Dynamic disturbance decoupling for nonlinear systems: the nonsquare and noninvertible case, in: B. Bonnard, B. Bride, J.P. Gauthier and I. Kupka, Eds., Analysis of controlled dynamical systems (Birkhäuser, Boston, 1991) 243-252.

[11] H.J.C. Huijberts, H. Nijmeijer and L.L.M. van der Wegen, Nonlinear dynamic disturbance decoupling, Proceedings $1990 \mathrm{CDC}$.

[12] S. Icart and J.F. Lafay, Design of a minimal precompensator for the decoupling problem, Systems Control Lett. 10 (1988) $325-332$.

[13] A. Isidori, Nonlinear Control Systems, Second Edition (Springer, Berlin, 1989).

[14] C.H. Moog, Nonlinear decoupling and structure at infinity, Math. Control Signals Systems 1 (1988) 257-268.

[15] H. Nijmeijer and W. Respondek, Dynamic input-output decoupling of nonlinear control Systems, IEEE Trans. Automat. Control 33 (1988) 1065-1070.

[16] H. Nijmeijer and A.J. van der Schaft, Nonlinear Dynamical Control Systems (Springer, New York, 1990).

[17] S.N. Singh, Decoupling of invertible nonlinear systems with state feedback and precompensation, IEEE Trans. Automat. Control 25 (1980) 1237-1239.

[18] S.N. Singh, A modified algorithm for invertibility in nonlinear systems, IEEE Trans. Automat. Control 26 (1981) 595-598.

[19] S.N. Singh, Generalised decoupled-control synthesis for invertible nonlinear systems, Proceedings IEEE D 128 (1981) $157-161$.

[20] L.L.M. Van der Wegen, Local Disturbance Decoupling with Stability for Nonlinear Systems, Lecture notes in control and information sciences, No. 166 (Springer, Berlin, 1991).

[21] S.H. Wang, Design of precompensator for decoupling problem, Electron. Lett. 6 (1970) 739-741.

[22] X.-H. Xia and W.-B. Gao, A minimal order compensator for decoupling a nonlinear system, Preprint (1989). 